\title{
Efficacy of twice-daily vs once-daily sessions of repetitive transcranial magnetic stimulation in the treatment of major depressive disorder: a retrospective study
}

This article was published in the following Dove Press journal:

Neuropsychiatric Disease and Treatment

\author{
Mandana Modirrousta ${ }^{1,2}$ \\ Benjamin P Meek $^{2}$ \\ Sara L Wikstrom ${ }^{2}$ \\ 'Department of Psychiatry, University \\ of Manitoba, ${ }^{2}$ Mental Health, Saint \\ Boniface General Hospital, Winnipeg, \\ MB, Canada
}

\begin{abstract}
Purpose: There is no clinical consensus on the optimal protocol for the treatment of major depressive disorder (MDD) using repetitive transcranial magnetic stimulation (rTMS). Accelerated protocols using more than a single session of treatment per day have been suggested as a means to reduce the overall length of time required for rTMS therapy. The objective of this study is to compare the treatment outcomes of patients with MDD who received two sessions of rTMS per day vs those who received one session per day, keeping the overall number of delivered pulses constant.
\end{abstract}

Patients and methods: In a retrospective study, we compared treatment outcomes of 36 patients with MDD who received 30 sessions of high-frequency $(10 \mathrm{~Hz}) \mathrm{rTMS}$ over the left dorsolateral prefrontal cortex. Patients received 3,000 pulses per session ( $5 \mathrm{~s}$ trains, $25 \mathrm{~s}$ intertrain interval) at $110 \%$ of resting motor threshold using a figure-eight coil. Patients received either two rTMS sessions per day $(n=17)$ or one session per day $(n=19)$. Depression symptoms were assessed by a psychiatrist using the Hamilton Rating Scale for Depression at baseline and after every 10 sessions of rTMS.

Results: The majority of patients in both groups responded to treatment, and there was a trend toward greater response rate in the twice-daily (TD) group (82.4\%) compared to the once-daily (OD) group (52.6\%). TD stimulation was tolerable for patients and produced no adverse side effects. Patients in the TD group experienced an improvement in symptoms faster than the OD group due to the accelerated therapy period.

Conclusion: Administration of two rTMS treatment sessions per day is tolerable for patients and does not seem to be inferior in efficacy to a OD protocol. TD administration has the benefit of producing symptom improvement over a shorter time span and requires fewer visits to the clinic.

Keywords: brain stimulation, affective disorders, treatment protocol, treatment effectiveness, accelerated protocol

\section{Introduction}

Transcranial magnetic stimulation (TMS) uses powerful (1.0-2.5 T), focused magnetic field pulses to non-invasively induce electrical currents in the neural tissue via an inductor coil placed against the scalp. ${ }^{1}$ Functional imaging studies have shown that high-frequency $(\mathrm{HF} ; \geq 5 \mathrm{~Hz}$ ) repetitive transcranial magnetic stimulation (rTMS) results in increased regional blood flow to the targeted area of the brain, whereas lowfrequency $(1 \mathrm{~Hz})$ rTMS has the opposite effect. ${ }^{2-4}$ This phenomenon can be exploited
Correspondence: Mandana Modirrousta St Boniface General Hospital, M4-McEwan Building, 363 Taché Ave, Winnipeg R2H 2A6, MB, Canada

Tel +l 2042372606

Fax + I 204233805 I

Email mmodirrousta@sbgh.mb.ca 
clinically to treat symptoms that result from localized cortical hypoactivity or hyperactivity. rTMS has become increasingly popular in recent decades as a therapy for treatment-resistant depression in part due to the absence of serious side effects, especially when compared to treatments such as electroconvulsive therapy (ECT). ${ }^{5}$ The therapeutic mechanism of clinical rTMS treatment likely relies on long-term potentiation and depression ${ }^{6}$ and appears to involve selective modulation of functional connectivity both within and between the central executive network and default mode network. ${ }^{7}$

Clinical trials and naturalistic studies have found that maximal therapeutic effects of rTMS treatment occur by 26-28 sessions of stimulation. ${ }^{8,9}$ For this reason, depression treatment in many clinics consists of 30 daily stimulation sessions administered over 6 weeks. rTMS is usually offered in an outpatient setting, meaning patients are required to make 30 separate visits to the rTMS clinic to complete a treatment. This time commitment may negatively influence treatment acceptance or completion in some cases. Indeed, treatment period has been identified as a general therapy-related factor affecting patients' compliance to treatment. ${ }^{10}$ Finding means of shortening the rTMS treatment period would not only reduce the time commitment required for rTMS but may also result in faster symptom improvement. This would have a positive impact on patients' well-being, facilitating an expedited return to their normal functioning.

Over the past decade, a number of groups have investigated the feasibility of accelerated rTMS treatment protocols. Protocols employing anywhere from 2 to 10 treatment sessions per day have been reported, ${ }^{11-16}$ indicating that patients can tolerate multiple sessions per day without serious side effects. Twice-daily (TD) treatment has been shown to be effective in the treatment of major depressive disorder (MDD) compared to sham, ${ }^{12,13}$ but evidence is lacking as to whether it is comparable in efficacy with once-daily (OD) protocols. Recently, Theleritis et $\mathrm{al}^{13}$ found that the odds of remission were marginally greater in a group of MDD patients receiving TD treatment compared to those receiving OD treatment. However, it is important to note that patients in the TD group received twice as many overall pulses as patients in the OD group, which might have contributed to the observed difference in response, and stimulation in this study was delivered at $100 \%$ of motor threshold (MT), which may be considered underpowered.

In addition to the number of sessions per day, the number of pulses per session and number of pulses per day have also been matters of interest with regards to both the safety and efficacy of rTMS treatment. Generally, due to safety concerns, most previous trials have limited the number of pulses administered per session to 3,000. ${ }^{17}$ However, higher doses have been utilized without adverse effect. For example, George et $\mathrm{al}^{18}$ have safely administered both 6,000 and 6,800 pulses per session using $10 \mathrm{~Hz}$ trains of $5 \mathrm{~s}$ with a 10 -s intertrain interval to 41 and 19 patients, respectively. ${ }^{19}$ The safety of large numbers of pulses per day has also been considered. Again, George et $\mathrm{al}^{18}$ have investigated a range of 12,960-18,000 pulses per day and reported minimal side effects with no serious adverse events. ${ }^{20}$ Nonetheless, accelerated rTMS protocols carry an increased risk of seizures and other side effects. As such, high-dose rTMS protocols should be investigated with great care to avoid unnecessary risk, with special attention paid to the length of interstimulus interval.

The effect of number of pulses per session and per day on treatment efficacy is inconclusive. Some meta-analyses have found no association between treatment outcome and number of pulses per day, ${ }^{21}$ whereas one meta-analysis demonstrated a negative correlation between treatment outcome and pulses per session, ${ }^{22}$ indicating that fewer pulses per session might be associated with a better antidepressant effect. Despite this overall effect, Kedzior et $\mathrm{al}^{22}$ suggest that certain patient subpopulations may benefit from different protocols, with initially nonresponsive individuals requiring more sessions with high numbers of pulses per session, while less treatment-resistant female patients may require fewer pulses per session. The heterogeneity of patient populations in most studies makes it difficult to tease apart the differential responses that specific patient clusters may have to various protocol parameters.

Here, we wanted to know whether an accelerated rTMS protocol using two treatment sessions per day would result in similar efficacy and acceptability to that of traditional OD treatment, controlling the overall number of pulses per treatment. By using a retrospective chart review, we compared treatment outcomes between patients who received OD treatment with those who received TD rTMS. To the extent of our knowledge, no study has directly compared the efficacy of OD to TD rTMS when using a protocol in which both groups received the same number of overall pulses. We hypothesized that a TD schedule would not produce additional adverse effects and would demonstrate similar treatment efficacy to an OD schedule.

\section{Patients and methods}

This study was approved by the University of Manitoba's Research Ethics Board. The rTMS clinic at St Boniface 
General Hospital maintains an anonymized record of the treatments administered on-site. This record includes TMS parameters and treatment outcomes, as well as basic demographic information and test scores associated with each treatment. This treatment record is maintained so that clinic efficacy can be regularly assessed by the TMS clinicians and protocols can be refined based on emerging clinical findings. The record contains no identifying information (eg, patient names, initials, health numbers, contact information). Given the anonymous nature of the rTMS record, the institutional review board did not require individual patient consent to access the records reviewed for this study. No identifying patient information was provided to the study authors during this review.

Protocol parameters and treatment outcomes were reviewed for all 36 patients with MDD who were treated with HF rTMS through the Neuromodulation and Neuropsychiatry Unit at St Boniface General Hospital between 2012 and 2016. Patients referred to this clinic were screened by a psychiatric nurse (SW) for the standard exclusion criteria for rTMS therapy: metal in the body, a family or personal history of epilepsy, brain tumor, or any other major neurological disorders. Patients were given a full psychiatric interview by the Unit psychiatrist (MM) before treatment, which included screening for disease-specific exclusion criteria such as psychotic depression, active suicidal ideations, dysthymia with no significant remission, and previous failure to ECT and/or rTMS therapy. All eligible candidates met Diagnostic and Statistical Manual of Mental Disorders, Fifth Edition (DSM-5) diagnostic criteria for $\mathrm{MDD}^{23}$ and did not have active substance use disorder or any past or current primary psychotic disorders. Patients scheduled to receive rTMS in this clinic were required to keep a stable medication regimen (no changes in medication or dosage) for 4 weeks before the start of rTMS and to maintain this stability for the duration of treatment.

Before the first rTMS session, patients completed a number of self-rated screening questionnaires including the Beck Depression Inventory (BDI-II), ${ }^{24}$ Beck Anxiety Inventory (BAI), ${ }^{25}$ Patient Health Questionnaire (PHQ-9), ${ }^{26}$ and Sheehan Disability Scale (SDS). ${ }^{27}$ In addition, the 17-item Hamilton Rating Scale for Depression (Ham-D) ${ }^{28}$ was administered by the treating psychiatrist (MM) within 1 week before the first session of rTMS and then again after every 10 sessions of treatment to monitor changes in symptoms. After 20 sessions, patients were discontinued from treatment if they showed no change (or a worsening) of Ham-D score from baseline.
All 36 patients received HF $10 \mathrm{~Hz}$ stimulation over the left dorsolateral prefrontal cortex (dlPFC) at 110\% of their resting MT. Resting MTs were established using the criterion of lowest intensity of stimulation that resulted in a visible movement of the dominant thumb (targeting the abductor pollicis brevis muscle) on 5 of 10 sequential pulses. MTs were not remeasured after the initial session, so each patient's treatment intensity remained the same throughout the length of their treatment. Treatments were administered daily, excepting weekends and holidays. Each HF treatment session consisted of a total of 3,000 TMS pulses delivered in 5-s trains with a 25-s intertrain interval.

Primary motor cortex and the treatment target area (left dlPFC) were co-registered with images from each individual's high-resolution three-dimensional T1-weighted magnetic resonance imaging (MRI) scans using the BrainSight ${ }^{\mathrm{TM}}$ 2 neuronavigator system (Rogue Research Inc., Montreal, QC, Canada). These images were normalized using MNI ATLAS coordinates and were superimposed with an overlay containing Brodmann areas (BAs). Stimulation was administered using a Magstim Rapid ${ }^{2}$ rTMS system with a figure-eight air-film coil (Magstim Co. Ltd., Whitland, UK) to stimulate the target area (BA 9 and 46).

For some patients $(n=10)$, the Brainsight neuronavigation system was not used to locate the dlPFC because the Brainsight equipment was not available in the clinic at the time of their treatments. For these patients, the " $5 \mathrm{~cm}$ rule" was used to locate the dlPFC ${ }^{29}$ by measuring $5 \mathrm{~cm}$ anterior along the scalp from the position that was found to be optimal for stimulation of the contralateral abductor pollicis brevis muscle during MT measurement.

The rTMS clinic at St Boniface General Hospital had three treatment slots available each day during the time covered by this review. As such, the clinic had the capacity to treat two patients per day, with one patient receiving a single stimulation session and the other receiving two sessions. In cases where patients received two sessions per day, sessions were performed consecutively separated by a 15-minute break. Patients were scheduled by the rTMS nurse to receive either one or two sessions of rTMS therapy per day based on whichever treatment slot was next available at the time of their acceptance. All patients received the same number of treatment sessions (30) and, therefore, the same number of overall pulses $(90,000)$ by the end of their treatment. However, patients on the OD schedule received their sessions once daily over 6 weeks, whereas patients on the TD schedule received their sessions twice daily over 3 weeks. rTMS was administered by the Unit nurse. 
For this review, treatment records were grouped based on whether the patient received one session (OD) or two sessions (TD) of rTMS per day. The primary outcome variable was treatment response, defined as a $\geq 50 \%$ drop in Ham-D score after the final session of treatment compared to baseline. Other outcomes of interest were change in Ham-D score across sessions and rates of remission. Remission was determined based on the results of a clinical interview with the Unit psychiatrist posttreatment and a final Ham-D score of less than seven.

Independent two sample $t$-tests were employed to compare the age and baseline clinical scores (Ham-D, BDI, BAI, PHQ, and SDS) of patients in the OD and TD groups, and a $z$-test was used to determine any difference between groups in the proportion of females. Percentage of responders and percentage of remitters after treatment were compared using $\chi^{2}$ tests. A repeated-measures analysis of variance (ANOVA) was used to compare symptom improvement between the two treatment protocols using Ham-D score across sessions (baseline, 10th session, 20th session, and 30th session) as the dependent variable and treatment type (OD vs TD) as the between-group factor. To assess whether demographic factors and baseline anxiety levels influenced symptom improvement, sex, age, and baseline BAI score were included as covariates in the repeated-measures ANOVA. Given a significant effect of session on Ham-D score, post hoc paired comparisons with Bonferroni correction were run to further elucidate the change in symptoms between the various assessments.

\section{Results}

Table 1 lists patients' demographic characteristics. There was no difference between the OD and TD groups in either the proportion of females $\left(\chi^{2}=0.000, p=0.986\right)$ or the age of patients $\left(\mathrm{t}_{34}=1.014, p=0.318\right)$. Ham-D scores at baseline were no different between the groups $\left(\mathrm{t}_{34}=0.531, p=0.599\right)$, indicating that patients' depression severity at baseline was

Table I Demographic information and baseline test scores

\begin{tabular}{lllll}
\hline & $\begin{array}{l}\text { OD group, } \\
\text { mean (SD) }\end{array}$ & $\begin{array}{l}\text { TD group, } \\
\text { mean (SD) }\end{array}$ & $\mathbf{t} / \chi^{2}$ & $p$-value \\
\hline Sex (female/male) & $10 / 9$ & $9 / 8$ & 0.000 & 0.986 \\
Age (years) & $50.6(I 5.5)$ & $45.4(14.5)$ & 1.014 & 0.318 \\
Ham-D baseline & $17.3(4.7)$ & $18.1(5.8)$ & 0.531 & 0.599 \\
BDI baseline & $37.4(4.7)$ & $38.4(10.4)$ & 0.312 & 0.759 \\
BAI baseline & $26.7(24.8)$ & $24.8(14.6)$ & 0.833 & 0.415 \\
PHQ baseline & $17.6(4.8)$ & $19.7(4.7)$ & 1.066 & 0.294 \\
SDS baseline & $21.6(6.2)$ & $21.4(4.7)$ & 0.080 & 0.937 \\
\hline
\end{tabular}

Abbreviations: BAI, Beck Anxiety Inventory; BDI, Beck Depression Inventory; Ham-D, Hamilton Rating Scale for Depression; OD, once-daily treatment; PHQ, Patient Health Questionnaire; SDS, Sheehan Disability Scale; TD, twice-daily treatment. similar in both groups. There was also no difference between groups in baseline scores on the BDI, BAI, PHQ, or SDS.

There was a general difference in the severity of depression at baseline as measured by the clinician-rated Ham-D scale vs the self-rated BDI scale. Mean Ham-D score across all patients $(\mathrm{M}=17.6, \mathrm{SD}=5.5)$ indicated more moderate depression, ${ }^{30}$ whereas mean BDI score $(\mathrm{M}=38.1, \mathrm{SD}=9.0)$ indicated more severe depression. ${ }^{31}$ A similar discrepancy has been noted in previous studies and found to be associated with lower age ( $<50$ years), higher education, high levels of neuroticism, and low levels of extraversion and agreeableness, as well as with an atypical, non-melancholic depressive subtype. ${ }^{32,33}$ Although our patient sample did not fall heavily into the $<50$-year-old age category $(\mathrm{M}=48.1, \mathrm{SD}=15.5)$, it is possible that some of these other factors played a role in this discrepancy. In addition, these two scales are known to emphasize different aspects of depression, so it is possible that our patient sample was biased toward more cognitive and affective elements of depression (emphasized by BDI) compared to more somatic and behavioral elements (emphasized by Ham-D). ${ }^{34}$

Across all treatments, mean motor threshold was $66.1 \%$ ( $\mathrm{SD}=7.9$ ) of stimulator output, resulting in a mean stimulator output of $72.4 \%$ ( $\mathrm{SD}=8.4$ ) for the treatment. Two patients from the OD group and one patient from the TD group were discontinued after their 20th session of treatment due to the lack of response. One responder in the OD group and one responder in the TD group quit at the end of their 22nd and 20 th sessions, respectively, for personal reasons. These five patients were included in our assessment of treatment response but excluded from subsequent repeated-measures ANOVAs due to missing 30th session Ham-D scores.

After the completion of rTMS treatment, $52.6 \%$ of patients in the OD group and $82.4 \%$ in the TD group were classified as responders based on $\mathrm{a} \geq 50 \%$ decrease in Ham-D score from baseline. This difference in response rate between treatment groups approached, but did not reach, significance (Pearson $\chi^{2}=3.49, p=0.062$ ). Remission rates showed a similar pattern in favor of TD treatment, but again these rates were not significantly different between groups $(\mathrm{OD}=36.8 \%$, $\left.\mathrm{TD}=64.7 \% ; \chi^{2}=2.80, p=0.095\right)$. Table 2 shows the response rate after 10,20 , and 30 sessions for each group.

Table 2 Response rate (\%) across treatment sessions

\begin{tabular}{llll}
\hline Session & $\mathbf{1 0}$ & $\mathbf{2 0}$ & $\mathbf{3 0}$ \\
\hline OD $(n=19)$ & 36.8 & 42.1 & 52.6 \\
TD $(n=17)$ & 29.4 & 70.6 & 82.4 \\
\hline
\end{tabular}

Abbreviations: OD, once-daily treatment; TD, twice-daily treatment. 
For patients who responded to treatment ( $\mathrm{n}=25 ; 11$ in the OD group and 14 in the TD group), we wanted to investigate whether there was a difference in the rate of symptom improvement across sessions depending on treatment protocol. To this end, we looked at the change in the Ham-D scores of responders across sessions. A repeated-measures ANOVA with Ham-D score (baseline, 10th session, 20th session, and 30th session) as the dependent variable and treatment type (OD vs TD) as the between-group factor revealed a significant main effect of session $\left(\mathrm{F}_{3,63}=90.766, p<0.001\right.$, $\left.\eta_{\mathrm{p}}^{2}=0.812\right)$ with no significant interaction effect $\left(\mathrm{F}_{3,63}=1.456\right.$, $\left.p=0.235, \eta_{\mathrm{p}}^{2}=0.069\right)$. Post hoc paired comparisons for repeated measures with Bonferroni correction showed that there was a significant change in Ham-D score after every 10 sessions ( $p<0.05$ for all pairwise comparisons). These results indicate that the responders in both groups showed a similar pattern of improvement after every 10 sessions. However, responders in the TD group achieved improvement in half the time of the OD responders (Figure 1) due to their accelerated treatment schedule.

To assess whether demographic factors and baseline anxiety levels influenced treatment response, sex, age, and baseline BAI score were included as covariates in the repeated-measures ANOVA. Sex $\left(\mathrm{F}_{1,20}=0.959, p=0.339\right.$, $\left.\eta_{\mathrm{p}}^{2}=0.045\right)$, age $\left(\mathrm{F}_{1,20}=0.244, p=0.626, \eta_{\mathrm{p}}^{2}=0.012\right)$, and BAI $\left(\mathrm{F}_{1,11}=0.001, p=0.979, \eta_{\mathrm{p}}^{2}=0.000\right)$ were found to have no influence on the treatment outcome, and there were no interaction effects between any of these variables and change in Ham-D score across time ( $\operatorname{sex}\left[\mathrm{F}_{1,60}=0.541, p=0.656\right.$, $\left.\eta_{\mathrm{p}}^{2}=0.026\right]$, age $\left[\mathrm{F}_{1,60}=0.107, p=0.956, \eta_{\mathrm{p}}^{2}=0.005\right]$, and BAI $\left.\left[\mathrm{F}_{1,33}=0.377, p=0.770, \eta_{\mathrm{p}}^{2}=0.033\right]\right)$.

To identify whether the use of the neuronavigator system had an effect on treatment outcome, we performed a covariate

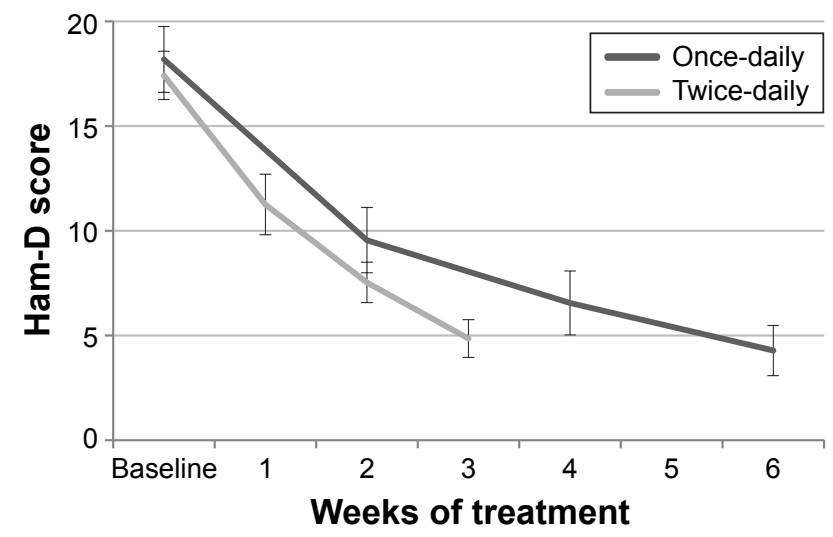

Figure I Change in Ham-D score throughout treatment for responders in the OD $(n=I I)$ and TD $(n=14)$ groups.

Abbreviations: Ham-D, Hamilton Rating Scale for Depression; OD, once-daily treatment; TD, twice-daily treatment. analysis using final Ham-D score as the between-group factor and use of the neuronavigator system (present or not present) as the covariate. The result showed no significant main effect, indicating that the presence of the neuronavigator system was not a contributing factor to treatment outcome $\left(\mathrm{F}_{1,29}=2.924\right.$, $p=0.098, \eta_{\mathrm{p}}^{2}=0.092$ ).

\section{Discussion}

This study produced two significant findings: 1) TD treatment was tolerable for patients with similar efficacy to that of OD application and 2) depressed patients in the TD group showed an improvement of clinical symptoms in half the time of those in the OD group.

It has been suggested that response to rTMS may be primarily related to the total number of magnetic pulses delivered. ${ }^{15}$ More recently, however, it has been proposed that the number of daily sessions over which pulses are administered may play a more significant role than the absolute number of pulses in treatment response.$^{35}$ Recently, Schulze et $\mathrm{a}^{36}$ presented a naturalistic case series of patients with MDD who received 6,000 pulses/day of bilateral dorsomedial prefrontal cortex rTMS split into either single-daily (ie, with no intersession interval) or two-daily sessions with a 1-hour intersession interval. The authors reported a significantly faster improvement rate in the twice-daily vs the once-daily group, suggesting that the number of sessions may be a more important factor than the number of total pulses per session for faster recovery.

In this study, the total number of pulses and the total number of sessions delivered were identical between the two groups, with the only difference being the number of treatment sessions that patients received per day. Although not significant, patients in the TD group showed a trend toward a greater response rate than those in the OD group. Kedzior et $\mathrm{a}^{22}$ suggested that certain patients (especially more treatment-resistant males) may benefit from more aggressive stimulation protocols. It is possible that an rTMS schedule employing more than one session per day may provide the extra stimulation required by some individuals to achieve treatment response. The fact that there was no difference between responders in the TD and OD groups in the rate of symptom improvement across sessions suggests that TD treatment is at least not inferior to a more traditional OD procedure and could feasibly be offered in cases where a TD schedule is more convenient either for the clinic or for the patient. That said, it is important not to overinterpret this negative finding, especially given the relatively low sample size considered in this study. 
An important consideration when administering multiple sessions of rTMS per day is the amount of rest time between sessions (intersession interval). It has been demonstrated that using theta-burst TMS as a priming stimulus 15 minutes before kinetic training can influence training outcome, ${ }^{37}$ suggesting that a 15 -minute pause may produce a facilitatory effect on subsequent stimulation. In contrast, recent functional magnetic resonance imaging (fMRI) studies have revealed that a break of at least 30 minutes between rTMS stimulation sessions may be required to produce neuroplastic effects in the second session. ${ }^{38}$ Thus, the 15 -minute break between sessions used to treat patients in this study may have been too short to attain the therapeutic potential of a TD protocol. More work is required to fully elucidate the optimal intersession interval for symptom improvement in rTMS schedules employing multiple daily sessions.

One major concern with accelerated rTMS protocols is patient safety. In this study, the protocol stimulation parameters for both groups fell within Rossi et al's ${ }^{39}$ safety recommendation guidelines. Patients in both groups tolerated treatment with no seizures or any other major side effects. Furthermore, clinical notes maintained by the rTMS nurse throughout the reviewed treatments revealed no difference in the severity or frequency of minor side effects - including headache and fatigue - between the two treatment groups. These results add to a growing literature demonstrating an absence of adverse consequences resulting from TD rTMS administration. ${ }^{11-13}$ Indeed, much more aggressive stimulation protocols have been used previously without issue, ${ }^{14-16,18,40}$ suggesting that humans can likely safely tolerate more intense treatment stimulation parameters than are currently recommended. Nonetheless, caution must be maintained, and patient safety held paramount as more aggressive protocols are explored. Incidents such as the depersonalization episode reported by Geerts et $\mathrm{al}^{41}$ after an accelerated rTMS program highlight the need for a cautious approach.

This study has a few main limitations. As a retrospective chart review, this analysis is limited due to the lack of a sham treatment control group as well as the fact that patients were not methodically randomized to a treatment arm, as would be the case in a clinical trial. This latter limitation is tempered by the fact that patients received either OD or TD purely based on rTMS clinic treatment slot availability; neither the treating psychiatrists nor the patients had any role in treatment selection. The other main limitation is that the raters in this study were not blind to the treatment the patients received, so it is possible that their ratings were affected by their knowledge of the treatment frequency. It should be noted, however, that the raters had no a priori hypotheses regarding treatment frequency; the decision to review these data was made at a time when all of the considered treatments and assessments had already been completed. Finally, the fact that different navigation methods were used to locate the treatment site adds heterogeneity to the reviewed records. Future clinical trials with controlled designs and different intersession intervals will be important in assessing the comparative efficacy of TD vs OD rTMS treatment.

\section{Conclusion}

Accelerated TD rTMS protocols for the treatment of MDD appear to be tolerable and safe to administer. In addition, a TD treatment schedule requires patients to make half as many visits to the clinic, and patients experience symptom improvement in a shorter time than a OD treatment. Future randomized control trials should further explore the feasibility and efficacy of rTMS schedules using multiple sessions per day. Special consideration will need to be given to the influence of intersession interval on treatment outcome. Electrophysiological and neuroimaging data will likely be invaluable in elucidating the ideal intersession interval for optimal neuroplastic effects. Given the significant social and economic disease burden of MDD, faster symptom improvement from accelerated rTMS protocols would be a boon for patients, their families, and health care providers.

\section{Acknowledgments}

This research was generously supported by the St Boniface Hospital Foundation. The abstract of this article was presented at the 2017 International Brain Stimulation Conference in Barcelona, Spain, as an abstract presentation with interim findings. The abstract was published in Brain Stimulation (DOI: http://dx.doi.org/10.1016/j.brs.2017.01.214).

\section{Disclosure}

The authors report no conflicts of interest in this work.

\section{References}

1. Hallett M. Transcranial magnetic stimulation: a primer. Neuron. 2007; 55(2):187-199.

2. Nahas Z, Lomarev M, Roberts DR, et al. Unilateral left prefrontal transcranial magnetic stimulation (TMS) produces intensity-dependent bilateral effects as measured by interleaved BOLD fMRI. Biol Psychiatry. 2001;50(9):712-720.

3. Fitzgerald PB, Sritharan A, Daskalakis ZJ, de Castella AR, Kulkarni J, Egan G. A functional magnetic resonance imaging study of the effects of low frequency right prefrontal transcranial magnetic stimulation in depression. J Clin Psychopharmacol. 2007;27(5):488-492.

4. Teneback CC, Nahas Z, Speer AM, et al. Changes in prefrontal cortex and paralimbic activity in depression following two weeks of daily left prefrontal TMS. J Neuropsychiatry Clin Neurosci. 1999;11(4):426-435. 
5. Ren J, Li H, Palaniyappan L, et al. Repetitive transcranial magnetic stimulation versus electroconvulsive therapy for major depression: a systematic review and meta-analysis. Prog Neuropsychopharmacol Biol Psychiatry. 2014;51:181-189.

6. Noda Y, Silverstein WK, Barr MS, et al. Neurobiological mechanisms of repetitive transcranial magnetic stimulation of the dorsolateral prefrontal cortex in depression: a systematic review. Psychol Med. 2015; 45(16):3411-3432.

7. Liston C, Chen AC, Zebley BD, et al. Default mode network mechanisms of transcranial magnetic stimulation in depression. Biol Psychiatry. 2014;76(7):517-526.

8. McDonald WM, Durkalski V, Ball ER, et al. Improving the antidepressant efficacy of transcranial magnetic stimulation: maximizing the number of stimulations and treatment location in treatment-resistant depression. Depress Anxiety. 2011;28(11):973-980.

9. Carpenter LL, Janicak PG, Aaronson ST, et al. Transcranial magnetic stimulation (TMS) for major depression: a multisite, naturalistic, observational study of acute treatment outcomes in clinical practice. Depress Anxiety. 2012;29(7):587-596.

10. Jin J, Sklar GE, Oh VMS, Li SH. Factors affecting therapeutic compliance: a review from the patient's perspective. Ther Clin Risk Manag. 2008;4(1):269-286.

11. McGirr A, Van den Eynde F, Tovar-Perdomo S, Fleck MP, Berlim MT. Effectiveness and acceptability of accelerated repetitive transcranial magnetic stimulation (rTMS) for treatment-resistant major depressive disorder: an open label trial. J Affect Disord. 2015;173:216-220.

12. Loo CK, Mitchell PB, McFarquhar TF, Malhi GS, Sachdev PS. A shamcontrolled trial of the efficacy and safety of twice-daily rTMS in major depression. Psychol Med. 2007;37(3):341-349.

13. Theleritis C, Sakkas P, Paparrigopoulos T, et al. Two versus one highfrequency repetitive transcranial magnetic stimulation session per day for treatment-resistant depression: a randomized sham-controlled trial. J ECT. 2017;33(3):190-197.

14. Desmyter S, Duprat R, Baeken C, Bijttebier S, van Heeringen K. The acute effects of accelerated repetitive transcranial magnetic stimulation on suicide risk in unipolar depression: preliminary results. Psychiatr Danub. 2014;26(suppl 1):48-52.

15. Holtzheimer PE, McDonald WM, Mufti M, et al. Accelerated repetitive transcranial magnetic stimulation for treatment-resistant depression. Depress Anxiety. 2010;27(10):960-963.

16. Baeken C, Marinazzo D, Wu GR, et al. Accelerated HF-rTMS in treatment-resistant unipolar depression: insights from subgenual anterior cingulate functional connectivity. World J Biol Psychiatry. 2014; 15(4):286-297.

17. Lefaucheur JP, André-Obadia N, Antal A, et al. Evidence-based guidelines on the therapeutic use of repetitive transcranial magnetic stimulation (rTMS). Clin Neurophysiol. 2014;125(11):2150-2206.

18. George MS, Raman R, Benedek DM, et al. A two-site pilot randomized 3 day trial of high dose left prefrontal repetitive transcranial magnetic stimulation (rTMS) for suicidal inpatients. Brain Stimul. 2014; 7(3):421-431.

19. Hadley D, Anderson BS, Borckardt JJ, et al. Safety, tolerability, and effectiveness of high doses of adjunctive daily left prefrontal repetitive transcranial magnetic stimulation for treatment-resistant depression in a clinical setting. J ECT. 2011;27(1):18-25.

20. Anderson B, Mishory A, Nahas Z, et al. Tolerability and safety of high daily doses of repetitive transcranial magnetic stimulation in healthy young men. J ECT. 2006;22(1):49-53.

21. Berlim MT, van den Eynde F, Tovar-Perdomo S, Daskalakis ZJ. Response, remission and drop-out rates following high-frequency repetitive transcranial magnetic stimulation (rTMS) for treating major depression: a systematic review and meta-analysis of randomized, double-blind and sham-controlled trials. Psychol Med. 2014;44(2): 225-239.
22. Kedzior KK, Azorina V, Reitz SK. More female patients and fewer stimuli per session are associated with the short-term antidepressant properties of repetitive transcranial magnetic stimulation (rTMS): a meta-analysis of 54 sham-controlled studies published between 1997-2013. Neuropsychiatr Dis Treat. 2014;10:727-756.

23. American Psychiatric Association. Diagnostic and Statistical Manual of Mental Disorders: DSM-5. 5th ed. Arlington, VA: American Psychiatric Association; 2013.

24. Beck AT, Steer RA, Ball R, Ranieri W. Comparison of beck depression inventories -IA and -II in psychiatric outpatients. J Pers Assess. 1996; 67(3):588-597.

25. Beck AT, Epstein N, Brown G, Steer RA. An inventory for measuring clinical anxiety: psychometric properties. J Consult Clin Psychol. 1988;56(6):893-897.

26. Kroenke K, Spitzer RL, Williams JB. The PHQ-9: validity of a brief depression severity measure. J Gen Intern Med. 2001;16(9):606-613.

27. Sheehan DV. The Anxiety Disease. New York, NY: Charles Scribners Sons; 1983.

28. Hamilton M. A rating scale for depression. J Neurol Neurosurg Psychiatry. 1960;23:56-62.

29. Pascual-Leone A, Rubio B, Pallardó F, Catalá MD. Rapid-rate transcranial magnetic stimulation of left dorsolateral prefrontal cortex in drug-resistant depression. Lancet. 1996;348(9022):233-237.

30. Zimmerman M, Martinez JH, Young D, Chelminski I, Dalrymple K. Severity classification on the Hamilton Depression Rating Scale. J Affect Disord. 2013;150(2):384-388.

31. Beck AT, Steer RA, Garbin MG. Psychometric properties of the Beck Depression Inventory: twenty-five years of evaluation. Clin Psychol Rev. 1988;8(1):77-100

32. Enns MW, Larsen DK, Cox BJ. Discrepancies between self and observer ratings of depression. The relationship to demographic, clinical and personality variables. J Affect Disord. 2000;60(1):33-41.

33. Schneibel R, Brakemeier EL, Wilbertz G, Dykierek P, Zobel I, Schramm E. Sensitivity to detect change and the correlation of clinical factors with the Hamilton Depression Rating Scale and the Beck Depression Inventory in depressed inpatients. Psychiatry Res. 2012;198(1):62-67.

34. Brown C, Schulberg HC, Madonia MJ. Assessing depression in primary care practice with the Beck Depression Inventory and the Hamilton Rating Scale for depression. Psychol Assess. 1995;7(1):59-65.

35. Downar J. Optimizing the inter-session interval for accelerated rTMS. Poster presented at the 2nd International Brain Stimulation Conference 2017; 2017; Barcelona, Spain.

36. Schulze L, Lozano C, Giacobbe P, et al. Number of pulses or number of sessions? Trajectories of improvement for once-daily vs. twice-daily dorsomedial prefrontal rTMS in major depression. Poster presented at the 2nd International Brain Stimulation Conference 2017; 2017; Barcelona, Spain.

37. Ackerley SJ, Stinear CM, Barber PA, Byblow WD. Combining theta burst stimulation with training after subcortical stroke. Stroke. 2010; 41(7):1568-1572.

38. Nettekoven C, Volz LJ, Kutscha M, et al. Dose-dependent effects of theta burst rTMS on cortical excitability and resting-state connectivity of the human motor system. J Neurosci. 2014;34(20):6849-6859.

39. Rossi S, Hallett M, Rossini PM, Pascual-Leone A. Safety, ethical considerations, and application guidelines for the use of transcranial magnetic stimulation in clinical practice and research. Clin Neurophysiol. 2009;120(12):2008-2039.

40. Baeken C, Vanderhasselt MA, Remue J, et al. Intensive HF-rTMS treatment in refractory medication-resistant unipolar depressed patients. J Affect Disord. 2013;151(2):625-631.

41. Geerts PJ, Lemmens GM, Baeken C. The occurrence of depersonalization symptoms after accelerated HF-rTMS of the left DLPFC in a patient with treatment-resistant depression: a case report. Brain Stimul. 2015;8(3):681-682. 


\section{Publish your work in this journal}

Neuropsychiatric Disease and Treatment is an international, peerreviewed journal of clinical therapeutics and pharmacology focusing on concise rapid reporting of clinical or pre-clinical studies on a range of neuropsychiatric and neurological disorders. This journal is indexed on PubMed Central, the 'PsycINFO' database and CAS,

and is the official journal of The International Neuropsychiatric Association (INA). The manuscript management system is completely online and includes a very quick and fair peer-review system, which is all easy to use. Visit http://www.dovepress.com/testimonials.php to read real quotes from published authors.

Submit your manuscript here: http://www.dovepress.com/neuropsychiatric-disease-and-treatment-journal 